\title{
Astronomical Science with Laser Guide Star Adaptive Optics: A Brief Review, a Current Snapshot, and a Bright Future
}

\author{
Michael C. Liu ${ }^{a}$ \\ ${ }^{a}$ Institute for Astronomy, University of Hawai'i, 2680 Woodlawn Drive, Honolulu, HI 96822; \\ USA
}

\begin{abstract}
We briefly discuss the past, present, and future state of astronomical science with laser guide star adaptive optics (LGS AO). We present a tabulation of refereed science papers from LGS AO, amounting to a total of 23 publications as of May 2006. The first decade of LGS AO science (1995-2004) was marked by modest science productivity ( $\approx 1$ paper/year), as LGS systems were being implemented and commissioned. The last two years have seen explosive science growth $(\approx 1$ paper/month), largely due to the new LGS system on the Keck II 10meter telescope, and point to an exciting new era for high angular resolution science. To illustrate the achievable on-sky performance, we present an extensive collection of Keck LGS performance measurements from the first year of our brown dwarf near-IR imaging survey. We summarize the current strengths and weaknesses of LGS compared to Hubble Space Telescope, offer a list of desired improvements, and look forward to a bright future for LGS given its wide-scale implementation on large ground-based telescopes.
\end{abstract}

Keywords: Adaptive optics, laser guide stars, high angular resolution, brown dwarfs, Keck Telescope

\section{INTRODUCTION}

Astronomers have envisioned using laser guide star adaptive optics (LGS AO) to achieve diffraction-limited observations from ground-based telescopes for over two decades. ${ }^{1-3}$ (See Refs. 4 and 5 for a historical review of LGS AO development.) The realization of these visions has been an arduous effort, but we are now entering a new epoch as LGS systems are commissioned on the largest ground-based telescopes. The scientific promise of near diffraction-limited imaging and spectroscopy from the ground over most of the sky is finally being realized.

At this key juncture, the purpose of this paper is to briefly review published LGS science to date; to provide a snapshot of the science that is being done with LGS AO; and to look ahead to a future path where LGS AO is a ubiquitous tool for observational astronomy.

\section{ASTRONOMICAL SCIENCE FROM LASER GUIDE STARS}

To date, four telecopes have produced astronomical science with LGS: the 1.5-meter Starfire Optical Range (SOR) Telescope in New Mexico; ${ }^{6}$ the 3 -meter Shane Telescope at Lick Observatory in California; ${ }^{7}$ the 3.5 -meter German-Spanish Astronomical Centre Telescope in Spain $;^{8}$ and the 10 -meter Keck II Telescope in Hawaii. ${ }^{9}$ The Starfire system used a Rayleigh-backscattered LGS, with the other systems using sodium laser guide stars.

The first refereed science paper from LGS AO was a study of the Orion Nebula region using the 1.5-meter telescope at Starfire Optical Range. ${ }^{10}$ As the authors pointed out: "it is a truism that if a new telescope or a new instrument can be pointed at [the Orion Nebula], it will be." They obtained $\mathrm{H} \alpha(6563 \AA)$ emission-line imaging of the photoevaporating circumstellar envelopes around the young stars. The poor natural seeing of the Starfire site and the low elevation of the Orion Nebula as viewed from New Mexico limited the AO system to only modest image correction, with image FWHMs of $0.4^{\prime \prime}$. Nevertheless, the LGS-sharpened images enabled detection of the cometary structure of these systems, demonstrating that good science opportunities can be harvested even when AO systems do not reach the diffraction limit.* The resolved circumstellar morphologies combined with

Email: mliu@ifa.hawaii.edu

*Indeed, the McCullough et al. (1995) work remains the most highly cited of all LGS papers to date, with 40 citations in ADS at the time this review was written, more than double the next most cited LGS paper. 

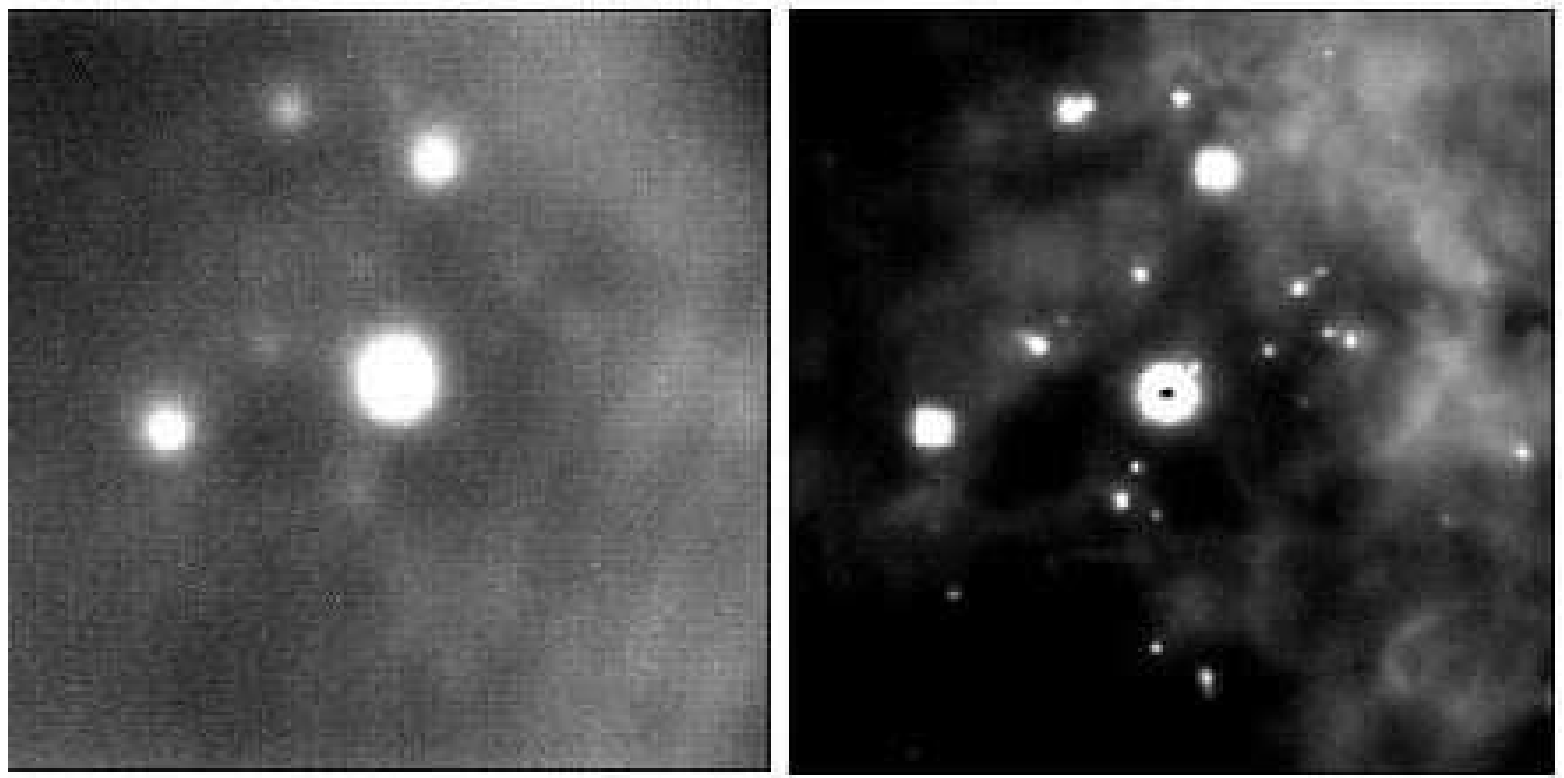

Figure 1. First astronomical science from LGS AO, optical $(0.65 \mu \mathrm{m})$ imaging of the central $40^{\prime \prime}$ of the Orion Nebula. ${ }^{10}$ The image of the left was obtained without AO and the one of the right with LGS AO. This figure was adapted from material on the Starfire Optical Range web site; see Ref. 10 for a higher quality reproduction.

quantitative modeling of the $\mathrm{H} \alpha$ and radio continuum fluxes supported the interpretation of these sources as undergoing photoevaporation due to the central massive star $\Theta^{1} \mathrm{C}$ Ori.

It is interesting to note that this first LGS science paper contained many of the characteristics and concerns that persist today for LGS astronomical science: (1) uncertainty in the PSF quality (see their Figure 3 for an analysis of anisoplanatism in their images); (2) using LGS photometry only for relative photometry of images, with absolute photometry derived from a seeing-limited dataset; (3) the need for modeling of the PSF (which was handled using multiple two-dimensional Gaussians), and (4) comparison with Hubble Space Telescope (HST) imaging results. ${ }^{11}$

Since the first paper, LGS science productivity has been relatively modest. As a point of comparison: as of July 2002, after about a decade of science operation, AO systems had produced 144 refereed science papers, nearly entirely derived from natural guide star (NGS) AO systems. ${ }^{12}$ A non-systematic search of the NASA Astrophysical Data System (ADS) abstract database indicates that the total number of AO science papers has about doubled since then, again dominated by NGS observations.

In comparison, LGS systems have produced 23 refereed science publications as of May 2006, again based on NASA ADS. The papers are listed in Table 1 and shown in Figure 1. This compilation includes only papers focused on astronomical science; AO instrumentation or "first light" papers are not included. The modest number of LGS AO papers compared to NGS AO is no doubt due to the vastly greater complexity and cost of LGS systems. Nevertheless, the sustained drive over many years for developing LGS AO points to the wide-spread appreciation for the science potential of this technology. The papers from 1995-2004, which were produced by the Starfire, Calar Alto, and Lick systems, can be categorized as:

- Binary field stars: 4 papers on resolved photometry and/or astrometry ${ }^{13-16}$

- Young stars: 3 papers on circumstellar material around pre-main sequence stars ${ }^{10,17,18}$

- Planetary astronomy: 2 papers on resolved imaging of asteroids or giant planets ${ }^{15,19}$

- Extragalactic science: 1 paper on the nuclear morphology of a barred spiral galaxy ${ }^{20}$ 


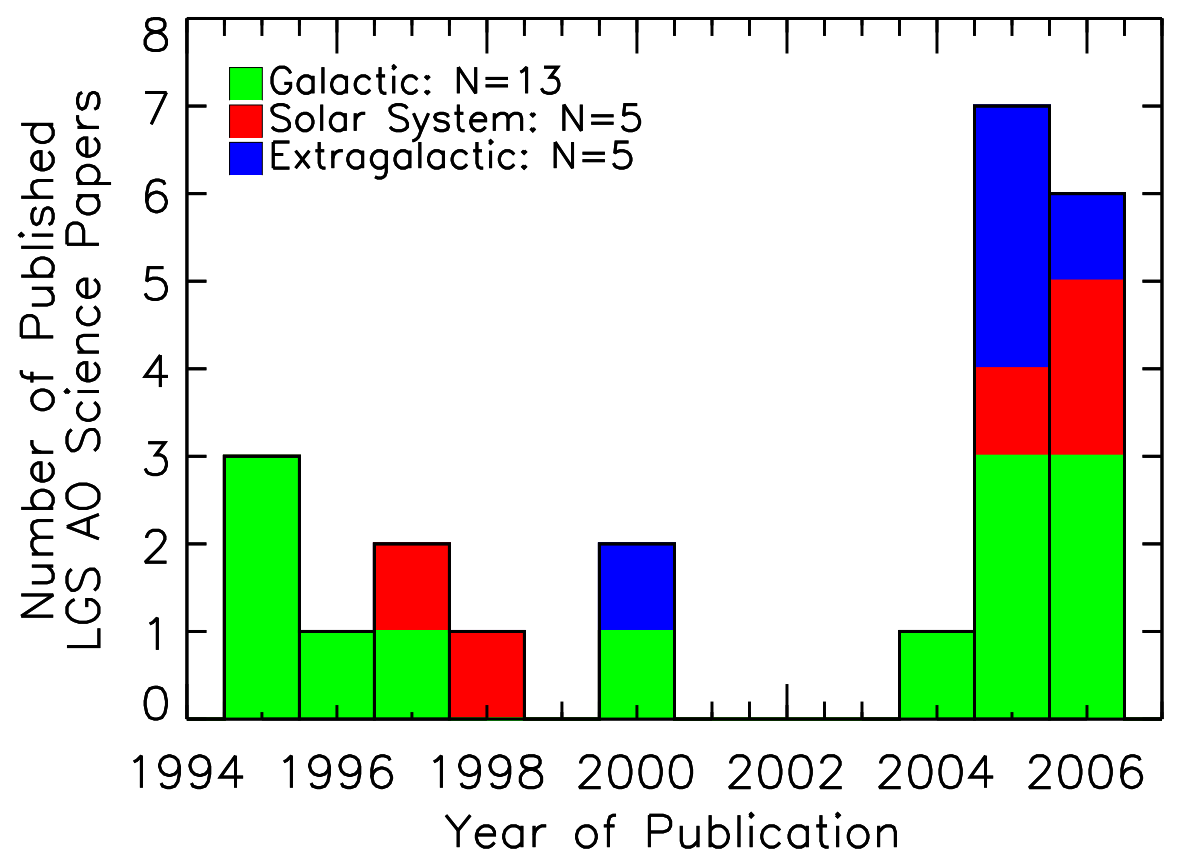

Figure 2. A histogram of all refereed LGS AO science papers published as of May 2006. The colors represent different science areas. The large spike in publications in 2005-2006 comes from the Keck LGS AO system.

A number of these papers used post-processing techniques, primarily deconvolution, to handle the spatially complex and time-variable PSF. Also, little extragalactic science was done; a significant limiting factor for LGS AO systems on $\sim 2-3$-meter telescopes is the need for relatively bright tiptilt stars, meaning very modest sky coverage.

The LGS AO system on the Keck II Telescope has been performing shared-risk science for about the past 1.5 years (since November 2004) and has produced all the LGS science papers in 2005-2006 so far. With its 10-meter aperture, Keck LGS can provide angular resolution far exceeding HST at IR wavelengths. In addition, the large aperture means the tiptilt star sensitivity $(R \lesssim 19)$ is sufficient to open most of the sky to LGS observations (see next section), which is a great boon for extragalactic science applications. There has been a substantial wave of initial science publications from Keck, which can be categorized into:

- Extragalactic science: 4 papers ${ }^{21-24}$

- Binary brown dwarfs: 3 papers ${ }^{25-27}$

- Galactic Center: 3 papers ${ }^{28-30}$

- Small bodies in the solar system: 3 papers ${ }^{31-33}$

The science breadth and impact of Keck LGS AO has been significant, including discovery of moons around small bodies in the solar system and the resulting mass measurements, ${ }^{31,33}$ color and variability measurements for Sgr A* in the Galactic Center, ${ }^{29,30}$ studying ultracool atmospheres with a new kind of binary brown dwarf, ${ }^{27}$ and decomposing the stellar components of $z \approx 0.5$ galaxies. ${ }^{21}$ As a point of reference, almost none of the initial Keck science results could have been accomplished with the previous generation of LGS AO systems. 
Table 1. Refereed science papers from LGS AO as of May 2006, listed by publication date. Some titles have been truncated and some title words abbreviated ("LGS" and "AO"). "Field" gives the area of study: "SS" = solar system, "Gal" = galactic, "Xgal" = extragalactic. " $N_{o b j}$ " indicates the number of science targets/fields observed with LGS.

\begin{tabular}{|c|c|c|c|c|c|c|}
\hline$\#$ & Authors, Journal & Title & Facilty & Field & $\lambda \lambda$ & $N_{o b j}$ \\
\hline 1 & $\begin{array}{l}\text { McCullough et al. } \\
1995, \text { ApJ }\end{array}$ & $\begin{array}{l}\text { Photoevaporating Stellar Envelopes Observed } \\
\text { with Rayleigh Beacon Adaptive Optics }\end{array}$ & SOR & Gal & $\mathrm{H} \alpha$ & 1 \\
\hline 2 & $\begin{array}{l}\text { Christou et al. } 1995, \\
\text { ApJ }\end{array}$ & $\begin{array}{l}\text { Rayleigh Beacon AO Imaging of ADS 9731: Mea- } \\
\text { surements of the Isoplanatic Field of View }\end{array}$ & SOR & Gal & $I J H$ & 1 \\
\hline 3 & $\begin{array}{l}\text { Drummond et al. } \\
1995, \mathrm{ApJ}\end{array}$ & $\begin{array}{l}\text { Full AO Images of ADS } 9731 \text { and } \mu \text { Cassiopeiae: } \\
\text { Orbits and Masses }\end{array}$ & SOR & Gal & $I J H$ & 1 \\
\hline 4 & $\begin{array}{l}\text { ten Brummelaar et } \\
\text { al. } 1996, \text { AJ }\end{array}$ & $\begin{array}{l}\text { Differential Binary Star Photometry Using the } \\
\text { AO System at Starfire Optical Range }\end{array}$ & SOR & Gal & $r i$ & 10 \\
\hline 5 & $\begin{array}{l}\text { Glenar et al. 1997, } \\
\text { PASP }\end{array}$ & $\begin{array}{l}\text { Multispectral Imagery of Jupiter and Saturn Us- } \\
\text { ing AO and Acousto-Optic Tuning }\end{array}$ & SOR & $\mathrm{SS}$ & $0.7-1.0 \mu \mathrm{m}$ & 2 \\
\hline 6 & $\begin{array}{l}\text { Koresko et al. 1997, } \\
\text { ApJ }\end{array}$ & $\begin{array}{l}\text { A Multiresolution Infrared Imaging Study of } \\
\mathrm{LkH} \alpha 198\end{array}$ & SOR & Gal & $H$ & 1 \\
\hline 7 & $\begin{array}{l}\text { Drummond et al. } \\
\text { 1998, Icarus }\end{array}$ & $\begin{array}{l}\text { Full AO Images of Asteroids Ceres and Vesta: Ro- } \\
\text { tational Poles \& Triaxial Ellipsoid Dimensions }\end{array}$ & SOR & $\mathrm{SS}$ & $i$ & 2 \\
\hline 8 & $\begin{array}{l}\text { Barnaby et al. 2000, } \\
\text { AJ }\end{array}$ & $\begin{array}{l}\text { Measurements of Binary Stars with the Starfire } \\
\text { Optical Range AO System }\end{array}$ & SOR & Gal & ri & 1 \\
\hline 9 & $\begin{array}{l}\text { Hackenberg et al. } \\
2000, \text { AA }\end{array}$ & $\begin{array}{l}\text { Near-Infrared AO Observations of Galaxy Clus- } \\
\text { ters: Abell } 262 \text { at } \mathrm{z}=0.0157 \ldots\end{array}$ & $\begin{array}{l}\text { Calar } \\
\text { Alto } \\
\end{array}$ & Xgal & $K$ & 1 \\
\hline 10 & $\begin{array}{l}\text { Perrin et al. 2004, } \\
\text { Science }\end{array}$ & $\begin{array}{l}\text { Laser Guide Star AO Imaging Polarimetry of Her- } \\
\text { big Ae/Be Stars }\end{array}$ & Lick & Gal & $J H K_{S}$ & 2 \\
\hline 11 & $\begin{array}{l}\text { Melbourne et al. } \\
\text { 2005, ApJL }\end{array}$ & $\begin{array}{l}\text { Merging Galaxies in GOODS-S: First Extragalac- } \\
\text { tic Results from Keck Laser AO }\end{array}$ & Keck & Xgal & $K^{\prime}$ & 1 \\
\hline 12 & $\begin{array}{l}\text { Gal-Yam et al. 2005, } \\
\text { ApJL }\end{array}$ & $\begin{array}{l}\text { A High Angular Resolution Search for the Pro- } \\
\text { genitor of the Type Ic Supernova } 2004 \mathrm{gt}\end{array}$ & Keck & Xgal & $K_{S}$ & 1 \\
\hline 13 & $\begin{array}{l}\text { Brown et al. 2005, } \\
\text { ApJL }\end{array}$ & $\begin{array}{l}\text { Keck LGS AO Discovery and Characterization of } \\
\text { a Satellite to Large Kuiper Belt Object } 2003 \text { EL61 }\end{array}$ & Keck & $\mathrm{SS}$ & $K^{\prime}$ & 1 \\
\hline 14 & $\begin{array}{l}\text { Muno et al. 2005, } \\
\text { ApJ }\end{array}$ & $\begin{array}{l}\text { A Remarkable Low-Mass X-ray Binary within } 0.1 \\
\text { pc of the Galactic Center }\end{array}$ & Keck & Gal & $K^{\prime} L^{\prime}$ & 1 \\
\hline 15 & Liu et al. 2005, ApJ & $\begin{array}{l}\text { Kelu-1 is a Binary L Dwarf: First Brown Dwarf } \\
\text { Science from Laser Guide Star AO }\end{array}$ & Keck & Gal & $J H K^{\prime}$ & 1 \\
\hline 16 & $\begin{array}{l}\text { Cohen et al. 2005, } \\
\text { ApJL }\end{array}$ & $\begin{array}{l}\text { To Be or Not to Be: Very Young Globular Clus- } \\
\text { ters in M31 }\end{array}$ & Keck & Xgal & $K^{\prime}$ & 6 \\
\hline 17 & Ghez et al. 2005, ApJ & $\begin{array}{l}\text { The First LGS AO Observations of the Galactic } \\
\text { Center: Sgr A*'s Infrared Color ... }\end{array}$ & Keck & Gal & $K^{\prime} L^{\prime}$ & 1 \\
\hline 18 & $\begin{array}{l}\text { Marchis et al. 2006, } \\
\text { Nature }\end{array}$ & $\begin{array}{l}\text { A Low Density of } 0.8 \mathrm{~g} \mathrm{~cm}^{-3} \text { for the Trojan Bi- } \\
\text { nary Asteroid } 617 \text { Patroclus }\end{array}$ & Keck & $\mathrm{SS}$ & $H K^{\prime}$ & 1 \\
\hline 19 & $\begin{array}{l}\text { Brown et al. 2006, } \\
\text { ApJ }\end{array}$ & Satellites of the Largest Kuiper Belt Objects & Keck & SS & $K^{\prime}$ & 4 \\
\hline 20 & $\begin{array}{l}\text { Krabbe et al. 2006, } \\
\text { ApJL }\end{array}$ & $\begin{array}{l}\text { Diffraction Limited Imaging Spectroscopy of the } \\
\text { SgrA* Region Using OSIRIS }\end{array}$ & Keck & Gal & $2.0-2.4 \mu \mathrm{m}$ & 1 \\
\hline 21 & $\begin{array}{l}\text { Gelino et al. 2006, } \\
\text { PASP }\end{array}$ & $\begin{array}{l}\text { Evidence of Orbital Motion in Binary Brown } \\
\text { Dwarf Kelu-1AB }\end{array}$ & Keck & Gal & $H K^{\prime}$ & 1 \\
\hline 22 & Liu et al. 2006, ApJ & $\begin{array}{l}\text { SDSS J1534+1615AB: A Novel T Dwarf Binary } \\
\text { Found with Keck LGS AO and the Role of Bina- } \\
\text { rity in the L/T Transition }\end{array}$ & Keck & Gal & $J H K^{\prime}$ & 1 \\
\hline 23 & $\begin{array}{l}\text { Sheehy et al. 2006, } \\
\text { ApJ }\end{array}$ & $\begin{array}{l}\text { Constraining the AO PSF in Crowded Fields: } \\
\text { Measuring Photometric Aperture Corrections }\end{array}$ & Keck & Xgal & $\bar{H}$ & 1 \\
\hline
\end{tabular}



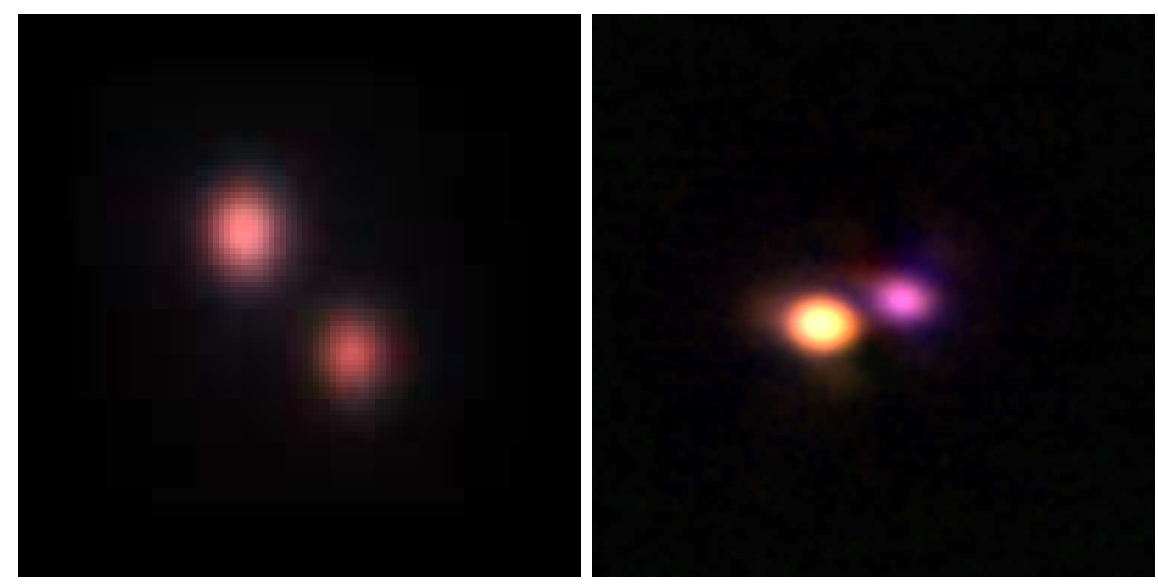

Figure 3. Two of the brown dwarf binaries discovered with Keck LGS AO, the L2+L4 binary Kelu-1AB (left) and the T1.5+T5.5 binary SDSS $1534+1615 \mathrm{AB}$ (right). ${ }^{25,27}$ The binaries have separations of $0.29^{\prime \prime}$ and $0.11^{\prime \prime}$, respectively. These color images were made from $J H K^{\prime}$-band data. The noticeably different colors of the two components of SDSS $1534+1615 \mathrm{AB}$ point to very different photospheric dust content, despite their similar masses and temperatures. ${ }^{27}$

\section{CURRENT KECK LGS PERFORMANCE: A NEAR-IR IMAGING SURVEY OF NEARBY BROWN DWARFS}

To illustrate what is currently possibly with LGS, we now discuss the on-sky performance of the Keck LGS system. For about the past year, my collaborators and I have been conducting a high angular resolution near-IR imaging survey of nearby brown dwarfs with the Keck LGS system (e.g., Figure 3). Our goals are (1) to assess the binary frequency of ultracool dwarfs; (2) to test atmospheric models with these coeval systems (e.g., as associated with the abrupt spectral transition from the L dwarfs to the T dwarfs); (3) to search for exceptionally low-temperature companions; and (4) to find and monitor substellar binaries suitable for dynamical mass determinations.

LGS AO represents a major instrumental advance for this science area. Brown dwarfs are too optically faint for natural guide star AO. Also, most known brown dwarf binaries have separations of $\lesssim 0.3^{\prime \prime},{ }^{34}$ hence the need for high angular resolution imaging to find and characterize them via resolved photometry and spectroscopy. Our observations have achieved $3-4 \times$ the angular resolution at $K$-band $(2.2 \mu \mathrm{m})$ compared to HST and thus are more sensitive to close companions. In addition, the ability of Keck LGS AO to find tighter binaries means that systems with much shorter orbital periods than the current sample can be found and expeditously monitored.

Our brown dwarf imaging survey provides an excellent dataset for assessing typical Keck LGS performance in the case of off-axis observations, namely the situation where the LGS is pointed to the science target but tiptilt sensing and correction are derived from an adjacent field star. Brown dwarfs are far too optically faint to serve as their own tiptilt references and hence the need for a nearby star - this is the same observing situation as expected for many extragalactic LGS applications and thus provides a good reference point. For Keck, the tiptilt star must be within $60^{\prime \prime}$ of the science target - in practice, we find that this results in a sky coverage fraction of about $2 / 3$ for an estimated $K$-band Strehl ratio of $\gtrsim 0.2$. Since wide-field brown dwarf searches encompass most of the sky (except for avoidance of the galactic plane), this $2 / 3$ sky coverage estimate is a fair representation of the fraction of any set of generic targets that can be imaged with LGS.

Figure 4 summarizes the quality of Keck LGS observations to date, based on multiple observing runs over the past year. No bad data have been censored, so a mix of seeing conditions and technical performance (e.g., LGS projected power and sodium light return flux) are represented. (See also paper 6272-01 by LeMignant et al. in this Proceedings.) The median $K$-band image FWHM for our survey is $0.069^{\prime \prime}$ with a best value of $0.049^{\prime \prime}$. The median Strehl is 0.18 with a best value of 0.43 . Good performance is achieved for tiptilt stars approaching $R \approx 18 \mathrm{mag}$, with best performance for $R \lesssim 17$ mag. We have successfully used tiptilt stars as widely separated as $60^{\prime \prime}$ from the science target, which represents the outer range of the tiptilt stage field of regard. 

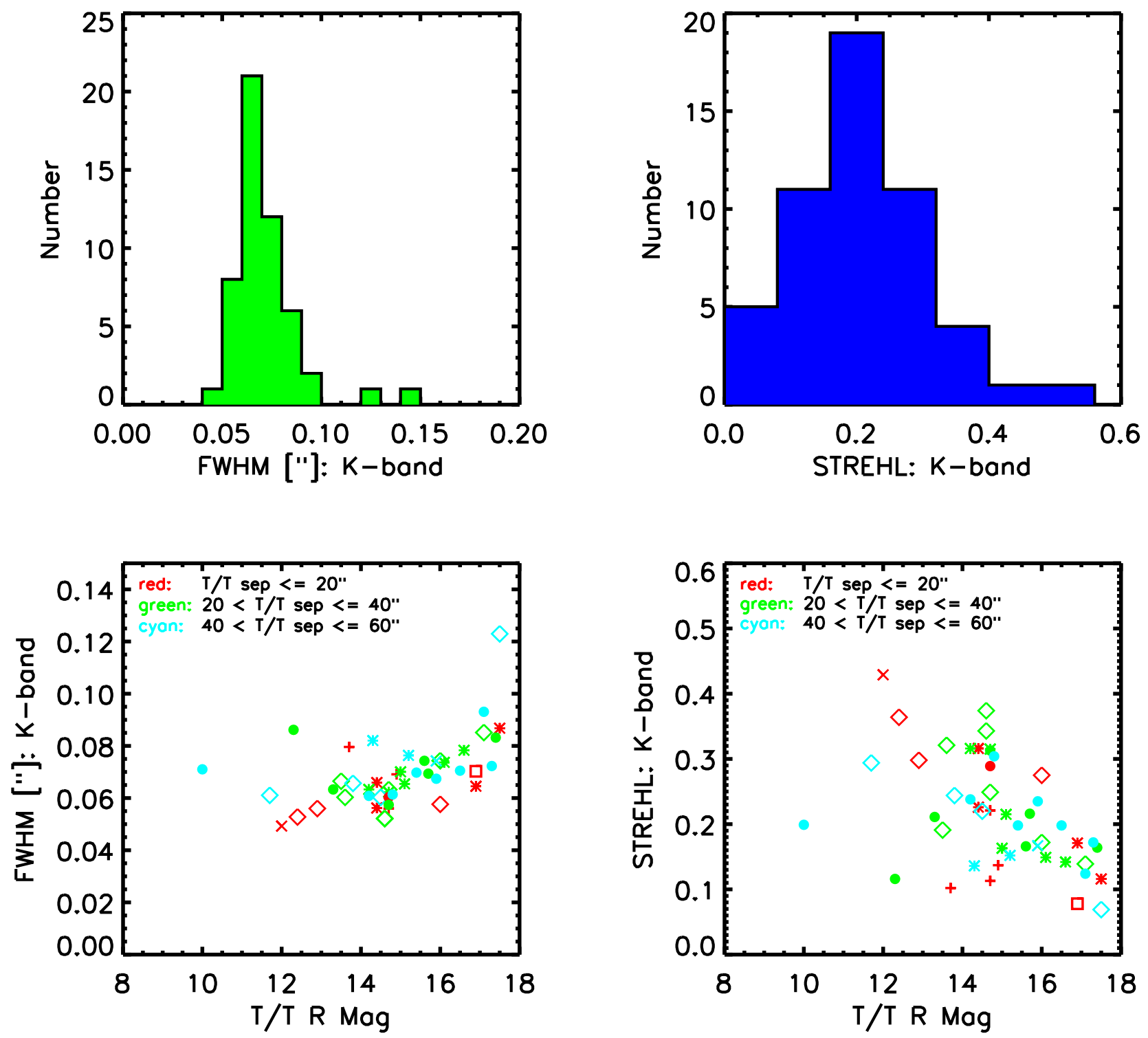

Figure 4. Summary of Keck LGS AO $K$-band $(2.2 \mu \mathrm{m})$ performance, based on our near-IR imaging survey of brown dwarfs. Each data point represents the average for a set of images of a given object. No bad data have been censored, so a mix of seeing conditions, target airmasses, and technical performance are represented. Top panels: Histogram of FWHM and Strehl ratios. The median FWHM is $0.069^{\prime \prime}$ with a best value of $0.049^{\prime \prime}$. The median Strehl is 0.18 with a best value of 0.43. Bottom panels: Image quality are a function of tiptilt star $R$-band magnitude, as listed in the USNO-B1.0 catalog. ${ }^{35}$ Different colors represent the angular separations of the tiptilt stars from the science targets, and different symbols represent different observing runs. 


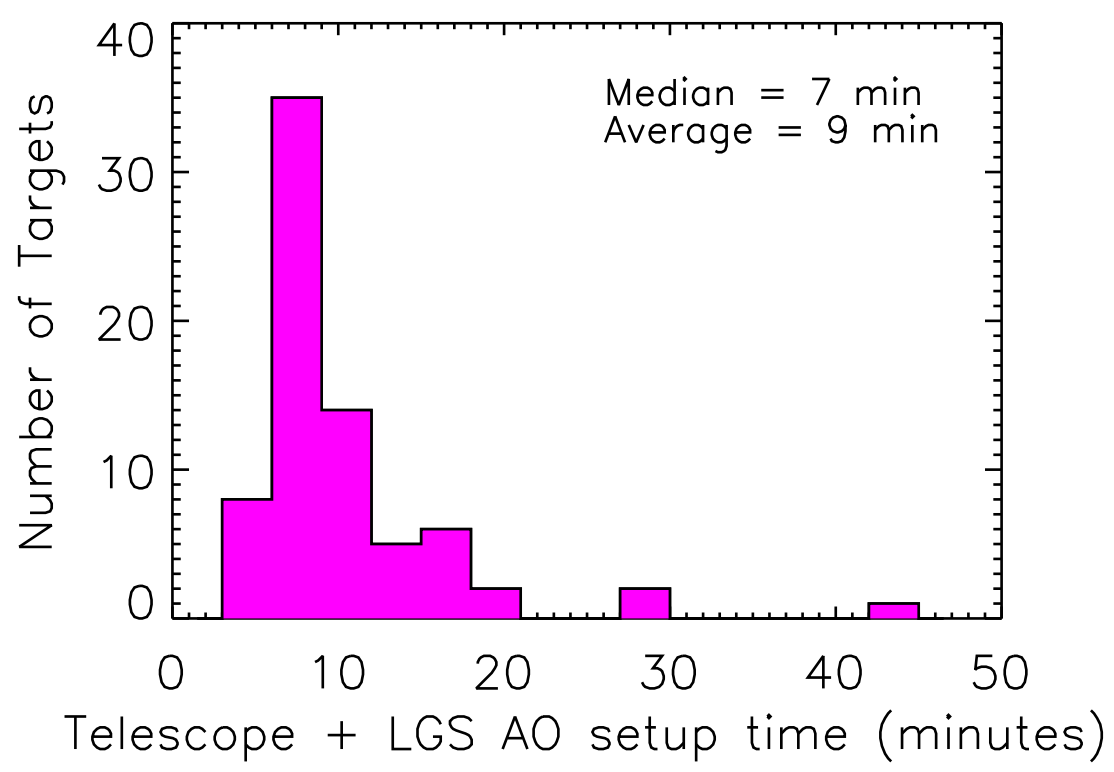

Figure 5. Target acquisition times for Keck LGS AO observing, from the first year of our brown dwarf imaging survey. These include telescope slew, field acquisition, LGS propagation, AO system optimization, and technical difficulties, but does not include the $\approx 30$ min needed at the start of each night for initial LGS AO setup.

\section{LASER GUIDE STARS VERSUS HUBBLE SPACE TELESCOPE}

The advent of LGS AO on 8-10 meter class telescopes naturally leads to the question of the relative roles of high angular resolution astronomy from the ground and from space. While implementation of LGS AO systems is ongoing, there are already several clear advantages of LGS compared to HST:

- Superior angular resolution in the near-IR: Keck LGS produces images as sharp as $0.05^{\prime \prime}$ FWHM at $H$ and $K$-bands, about $3-4 \times$ better than HST. In general, LGS AO excels at studying close point sources, such as tight binaries ${ }^{25-27,31-33}$ and dense star clusters. ${ }^{24,29}$ LGS is likely to become a popular platform for these kinds of near-IR observations. In addition, LGS in the near-IR offers comparable angular resolution to HST at optical wavelengths, allowing for well-matched imaging studies over a wide-range of wavelengths. ${ }^{21,22}$

- Ability to obtain thermal IR imaging $(3-4 \mu \mathrm{m})$ and spatially resolved IR $(1-5 \mu \mathrm{m})$ spectroscopy: ${ }^{28-30}$ Neither of these capabilities is available from HST.

- Efficient survey-style observing: LGS AO can observe many (>20) targets in one night, whereas HST is limited to about one target per orbit. Thus, LGS AO can carry out high angular resolution surveys of many objects, which would otherwise require a prohibitive number of HST observing time. Figure 5 shows the target acquisition times experienced by our Keck LGS survey, including telescope slew, LGS propagation, and $\mathrm{AO}$ performance optimization. The average setup time is 9 minutes, with times as short as 5 minutes and a long tail in the distribution due to occasional significant technical problems.

- Long-term availability: LGS AO offers an enduring platform for multi-epoch high angular resolution science, e.g., monitoring of time-variable phenomena or orbital motion. ${ }^{\dagger}$ In contrast, the long-term future is $H S T$ is uncertain.

\footnotetext{
${ }^{\dagger}$ An interesting illustration of this is provided by the case of Kelu-1. One of the first free-floating brown dwarfs discovered in the solar neighborhood, ${ }^{36}$ this nearby L dwarf had long been noted to be overluminous compared to other similar objects. ${ }^{37,38}$ HST imaging in 1998 failed to identify any close companion, ${ }^{39}$ leading to the intriguing possibility that Kelu-1's high luminosity was due to a very young age ( $\sim 10 \mathrm{Myr})$; the implied mass would have been only $\approx 12 \mathrm{M}_{\mathrm{Jup}}{ }^{40}$ Keck LGS imaging in 2005 discovered Kelu-1 to be a $0.29^{\prime \prime}$ binary and showed that binarity explains its many anomalous properties (high luminosity, very red color, high inferred effective temperature, and low lithium absorption). ${ }^{25}$ The
} 
- Novel instrumentation tailored for LGS AO: Examples here include Keck's near-IR integral field spectrograph OSIRIS ${ }^{41}$ and the dual-channel imaging polarimeter at Lick Observatory. ${ }^{18}$

And the current disadvantages of LGS compared to HST include:

- Limited to the IR wavelengths: LGS AO offers best performance at $H$-band $(1.6 \mu \mathrm{m})$ and longer wavelengths. High angular resolution imaging at optical wavelengths remains the domain of HST.

- Complex point spread function (PSF): The LGS AO PSF is irregular in appearance and varies in time. Therefore, in contrast to imaging of point sources, LGS studies of extended sources ("fuzzy blob science") is especially challenged by the uncertainties in the PSF at the time of observation. This is also true for studies of very faint point sources next to much brighter point sources, where image contrast is the key requirement rather than pure angular resolution. In these cases, the exact dividing line between HST and LGS AO will depend on the particular science goals.

- Limited field of view: Good LGS correction is restricted to the isoplanatic angle, $\approx 30-40^{\prime \prime}$ at $K$-band, and the PSF is spatially variable across this field.

- Need for a tiptilt star: Only about 2/3 of the sky is accessible to LGS AO, due to the need for the tiptilt star close to the science target - specific objects of interest may only be observable by HST. This sky coverage fraction is an approximate average over the entire sky; LGS coverage will be worse near the galactic poles (e.g., the Hubble Deep Field) and better close to the galactic plane.

- Heterogenous data: LGS AO performance can vary greatly, as it depends on seeing conditions, laser+AO performance, and tiptilt star properties (Figure 4). HST offers much more predictable performance.

\section{CONCLUDING THOUGHTS: EXPECTATIONS AND WISHES FOR THE (NOT-TOO-DISTANT) FUTURE}

More than two decades since its conception, LGS AO is now entering a new phase in its growth, opening a new era in high angular resolution science. The first decade of astronomical science was marked by modest science productivity, as these LGS AO systems were commissioned and optimized $(\approx 1$ science paper/year). Benefiting from the fruits of this effort, the LGS AO system on the Keck II 10-meter telescope has had a highly successful first 1.5 years of science $(\approx 1$ paper/month). Several LGS systems are planned to come online in the next two years, including those at the Gemini-North (2006), VLT (2006), Palomar 5-meter (2006), MMT (2006), Subaru (2007), Gemini-South (2007), and the Keck I (2008) telescopes. Given the size and quality of the science communities associated with these new AO systems, we can look forward to even more significant growth in LGS science soon $(\approx 1$ paper/week?).

We conclude with a non-comprehensive wishlist for future LGS developments, in order to highlight ongoing efforts and to review some outstanding challenges:

- Field of view: Single LGS systems produce a corrected field of view the size of the isoplanatic angle. In fact, nearly all LGS science thus far has been restricted to objects that span $\lesssim 10^{\prime \prime}$ across (and most typically $\left.\lesssim 2^{\prime \prime}\right)$. The upcoming Gemini-South multi-conjugate AO system is notable, as it will be the first LGS AO system to correct much larger fields of view $\left(\approx 1-2^{\prime}\right)$.

- Observing efficiency: Nighttime LGS AO operations currently require many more personnel compared to regular seeing-limited observations. (See also paper 6270-12 by LeMignant et al. in this Proceedings.) Greater automation will reduce this burden on telescope staff and also should lead to greater efficiency, as observing procedures are streamlined. For "survey-style" science programs, in some sense the science return is proportional to the number of targets observed; therefore more efficient observing is a significant benefit.

projected separation of the binary was too small to be easily resolved at the time of the original $H S T$ imaging, ${ }^{26}$ but subsequent orbital motion over 7 years enabled the binary to be found with Keck LGS AO. 
- Robust real-time performance predictions: LGS image quality varies depending on nightly weather conditions, laser performance, AO performance, sodium layer density and structure, and tiptilt star properties. To maximize the science return, it would be desireable to be able to robustly predict LGS performance for any given target on any given night. This capability would allow observations to be tailored to achieve the desired science goal. Queue-scheduled observing is a key element here, but also better real-time understanding of seeing conditions, LGS performance and the conditions in the sodium layer are need.

- Near-IR tiptilt sensors: While much of the sky is available for LGS, the most obscured regions (e.g., starforming regions) are not due to the lack of optically visible tiptilt stars. Tiptilt sensors working at near-IR wavelengths would open the door to studying the youngest stages of star and planet formation.

- High quality catalogs for tiptilt stars: Our brown dwarf imaging survey finds about 1 in 10 tiptilt stars with $R \lesssim 18$ mag from the USNO-B1.0 catalog ${ }^{35}$ are unsuitable, either because they turn out to be faint galaxies or they turn out to be much fainter than the reported magnitudes. The Pan-STARRS project ${ }^{42}$ will provide precise, multi-band photometry over the entire sky visible from Hawaii, with the initial PS-1 telescope beginning operations later this year. Combined with the SkyMapper Telescope ${ }^{43}$ in the southern hemisphere, high quality all-sky catalogs should be available in a few years for robust selection of tiptilt stars.

- Improved PSF stability and characterization: The time-variability of the LGS PSF will remain a significant concern for the foreseeable future. Post-processing software techniques can provide some immediate assistance, e.g., deconvolution and/or PSF modeling techniques. ${ }^{24,44,45}$ Development of algorithms to use AO telemetry data to estimate the real-time PSF would be a valuable capability for LGS. ${ }^{46}$ On a somewhat longer timescale, instruments tailored to handle the challenges of LGS AO imaging, such as dual-channel imaging systems, can circumvent this problem for some types of science programs. Finally, next-generation LGS AO systems should produce higher Strehl imaging, leading to more stable and well-behaved PSFs.

While the technology is far from mature, LGS AO is entering a phase of rapid growth. There is little doubt that it will quickly become a key capability for a very broad range of astrophysics, spanning the nearest solar system bodies to the highest redshift galaxies and the entire universe in-between.

\section{ACKNOWLEDGMENTS}

It is pleasure to thank the many, many, many people who have imagined, advocated, designed, constructed, tested, commissioned, supported, and/or persevered to make astronomy with laser guide stars a reality. We also gratefully acknowledge the teams at Laurence Livermore National Laboratory, Lick Observatory, and Keck Observatory for stoking our enthusiasm for LGS AO. We thank Dagny Looper for the Keck LGS performance analysis, Peter Wizinowich for providing the Keck LGS bibliography, and several colleagues for proofreading the contents of Table 1. MCL's research presented herein is partially supported from NSF grant AST-0507833 and an Alfred P. Sloan Research Fellowship. We wish to recognize and acknowledge the very significant cultural role and reverence that the summit of Mauna Kea has always had within the indigenous Hawaiian community. We are most fortunate to have the opportunity to conduct observations from this mountain.

\section{REFERENCES}

1. R. Foy and A. Labeyrie, "Feasibility of adaptive telescope with laser probe," $A \mathscr{E} A$ 152, pp. L29-L31, Nov. 1985.

2. L. A. Thompson and C. S. Gardner, "Experiments on laser guide stars at Mauna Kea Observatory for adaptive imaging in astronomy," Nature 328, pp. 229-231, July 1987.

3. W. Happer, G. J. MacDonald, C. E. Max, and F. J. Dyson, "Atmospheric-turbulence compensation by resonant optical backscattering from the sodium layer in the upper atmosphere," Optical Society of America Journal A 11, pp. 263-276, Jan. 1994.

4. F. Roddier, Adaptive Optics in Astronomy, Adaptive Optics in Astronomy, Edited by François Roddier, pp. 419. ISBN 0521612144. Cambridge, UK: Cambridge University Press, November 2004., Nov. 2004. 
5. J. W. Hardy, ed., Adaptive optics for astronomical telescopes, 1998.

6. R. Q. Fugate, "Two generations of laser-guide-star adaptive-optics experiments at the Starfire Optical Range," Optical Society of America Journal A 11, pp. 310-324, 1994.

7. C. E. Max et al., "Image improvement from a sodium-layer laser guide star adaptive optics system," Science 277, p. 1649, 1997.

8. A. Eckart et al., "ALFA: The MPIA/MPE Laser Guide Star AO System," Experimental Astronomy 10, pp. 1-3, Apr. 2000.

9. P. L. Wizinowich et al., "The W. M. Keck Observatory Laser Guide Star Adaptive Optics System: Overview," PASP 118, pp. 297-309, Feb. 2006.

10. P. R. McCullough, R. Q. Fugate, J. C. Christou, B. L. Ellerbroek, C. H. Higgins, J. M. Spinhirne, R. A. Cleis, and J. F. Moroney, "Photoevaporating Stellar Envelopes Observed with Rayleigh Beacon Adaptive Optics," ApJ 438, pp. 394-403, Jan. 1995.

11. C. R. O'Dell, Z. Wen, and X. Hu, "Discovery of new objects in the Orion nebula on HST images - Shocks, compact sources, and protoplanetary disks," ApJ 410, pp. 696-700, June 1993.

12. L. M. Close, "A Review of Published Adaptive Optics Science: A Bright Future for Adaptive Optics," in Discoveries and Research Prospects from 6- to 10-Meter-Class Telescopes II. Edited by Guhathakurta, Puragra. Proceedings of the SPIE, Volume 4834, pp. 84-100 (2003)., P. Guhathakurta, ed., pp. 84-100, Feb. 2003.

13. J. C. Christou, B. Ellerbroek, R. Q. Fugate, D. Bonaccini, and R. Stanga, "Rayleigh Beacon Adaptive Optics Imaging of ADS 9731: Measurements of the Isoplanatic Field of View," ApJ 450, p. 369, Sept. 1995.

14. T. A. T. Brummelaar, B. D. Mason, W. G. Bagnuolo, W. I. Hartkopf, H. A. McAlister, and N. H. Turner, "Differential Binary Star Photometry Using the Adaptive Optics System at Starfire Optical Range," AJ 112, p. 1180, Sept. 1996.

15. J. D. Drummond, R. Q. Fugate, J. C. Christou, and E. K. Hege, "Full Adaptive Optics Images of Asteroids Ceres and Vesta; Rotational Poles and Triaxial Ellipsoid Dimensions," Icarus 132, pp. 80-99, Mar. 1998.

16. D. Barnaby, E. Spillar, J. C. Christou, and J. D. Drummond, "Measurements of Binary Stars with the Starfire Optical Range Adaptive Optics Systems," AJ 119, pp. 378-389, Jan. 2000.

17. C. D. Koresko, P. M. Harvey, J. C. Christou, R. Q. Fugate, and W. Li, "A Multiresolution Infrared Imaging Study of LkH $\alpha$ 198," ApJ 485, p. 213, Aug. 1997.

18. M. D. Perrin, J. R. Graham, P. Kalas, J. P. Lloyd, C. E. Max, D. T. Gavel, D. M. Pennington, and E. L. Gates, "Laser Guide Star Adaptive Optics Imaging Polarimetry of Herbig Ae/Be Stars," Science 303, pp. 1345-1348, Feb. 2004.

19. D. A. Glenar, J. J. Hillman, M. Lelouarn, R. Fugate, and J. D. Drummond, "Multispectral Imagery of Jupiter and Saturn Using Adaptive Optics and Acousto-Optic Tuning," PASP 109, pp. 326-337, Mar. 1997.

20. W. Hackenberg, A. Eckart, R. I. Davies, S. Rabien, T. Ott, M. Kasper, S. Hippler, and A. Quirrenbach, "Near-infrared adaptive optics observations of galaxy clusters: Abell 262 at z=0.0157, J1836.3CR at z $=0.414$, and PKS 0743-006 at z=0.994," A\&A 363, pp. 41-61, Nov. 2000.

21. J. Melbourne et al., "Merging Galaxies in GOODS-S: First Extragalactic Results from Keck Laser Adaptive Optics," ApJ 625, pp. L27-L30, May 2005.

22. A. Gal Yam, D. B. Fox, S. R. Kulkarni, K. Matthews, D. C. Leonard, D. J. Sand, D.-S. Moon, S. B. Cenko, and A. M. Soderberg, "A High Angular Resolution Search for the Progenitor of the Type Ic Supernova 2004gt," ApJ 630, pp. L29-L32, Sept. 2005.

23. J. G. Cohen, K. Matthews, and P. B. Cameron, "To Be or Not to Be: Very Young Globular Clusters in M31," ApJ 634, pp. L45-L48, Nov. 2005.

24. C. D. Sheehy, N. McCrady, and J. R. Graham, "Constraining the Adaptive Optics Point-Spread Function in Crowded Fields: Measuring Photometric Aperture Corrections," ArXiv Astrophysics e-prints , Apr. 2006.

25. M. C. Liu and S. K. Leggett, "Kelu-1 is a Binary L Dwarf: First Brown Dwarf Science from Laser Guide Star Adaptive Optics," ApJ 634, p. 616, 2005.

26. C. R. Gelino, S. R. Kulkarni, and D. C. Stephens, "Evidence of Orbital Motion in the Binary Brown Dwarf Kelu-1AB," PASP 118, pp. 611-616, Apr. 2006. 
27. M. C. Liu, S. K. Leggett, D. A. Golimowski, K. Chiu, X. Fan, T. R. Geballe, D. P. Schneider, and J. Brinkmann, "SDSS J1534+1615AB: A Novel T Dwarf Binary Found with Keck Laser Guide Star Adaptive Optics and the Potential Role of Binarity in the L/T Transition," ArXiv Astrophysics e-prints, May 2006.

28. M. P. Muno et al., "A Remarkable Low-Mass X-Ray Binary within 0.1 Parsecs of the Galactic Center," ApJ 633, pp. 228-239, Nov. 2005.

29. A. M. Ghez et al., "The First Laser Guide Star Adaptive Optics Observations of the Galactic Center: Sgr A*'s Infrared Color and the Extended Red Emission in its Vicinity," ApJ 635, pp. 1087-1094, Dec. 2005.

30. A. Krabbe, C. Iserlohe, J. E. Larkin, M. Barczys, M. McElwain, J. Weiss, S. A. Wright, and A. Quirrenbach, "Diffraction-limited Imaging Spectroscopy of the Sagittarius A* Region Using OSIRIS, a New Keck Instrument," ApJ 642, pp. L145-L148, May 2006.

31. M. E. Brown et al., "Keck Observatory Laser Guide Star Adaptive Optics Discovery and Characterization of a Satellite to the Large Kuiper Belt Object 2003 EL_61," ApJ 632, pp. L45-L48, Oct. 2005.

32. F. Marchis et al., "A low density of $0.8 \mathrm{~g} \mathrm{~cm}^{-3}$ for the Trojan binary asteroid 617 Patroclus," Nature 439, pp. 565-567, Feb. 2006.

33. M. E. Brown et al., "Satellites of the Largest Kuiper Belt Objects," ApJ 639, pp. L43-L46, Mar. 2006.

34. A. J. Burgasser, I. N. Reid, N. Siegler, L. Close, P. Allen, P. Lowrance, and J. Gizis, "Not Alone: Tracing the Origins of Very Low Mass Stars and Brown Dwarfs Through Multiplicity Studies," ArXiv Astrophysics e-prints , Feb. 2006.

35. D. G. Monet et al., "The USNO-B Catalog," AJ 125, pp. 984-993, Feb. 2003.

36. M. T. Ruiz, S. K. Leggett, and F. Allard, "Kelu-1: A Free-floating Brown Dwarf in the Solar Neighborhood," ApJ 491, p. L107, Dec. 1997.

37. E. L. Martín, X. Delfosse, G. Basri, B. Goldman, T. Forveille, and M. R. Zapatero Osorio, "Spectroscopic Classification of Late-M and L Field Dwarfs," AJ 118, pp. 2466-2482, Nov. 1999.

38. S. K. Leggett et al., "Infrared Photometry of Late-M, L, and T Dwarfs," ApJ 564, p. 452, 2002.

39. E. L. Martín, G. Basri, W. Brandner, J. Bouvier, M. R. Zapatero Osorio, R. Rebolo, J. Stauffer, F. Allard, I. Baraffe, and S. T. Hodgkin, "Discovery of a Very Low Mass Binary with the Hubble Space Telescope Near-Infrared Camera and Multiobject Spectrometer," ApJ 509, pp. L113-L116, Dec. 1998.

40. D. A. Golimowski et al., "L' and M' Photometry of Ultracool Dwarfs," AJ 127, pp. 3516-3536, June 2004.

41. J. E. Larkin et al., "OSIRIS: infrared integral field spectrograph for the Keck adaptive optics system," in Instrument Design and Performance for Optical/Infrared Ground-based Telescopes. Edited by Iye, Masanori; Moorwood, Alan F. M. Proceedings of the SPIE, Volume 4841, pp. 1600-1610 (2003)., M. Iye and A. F. M. Moorwood, eds., pp. 1600-1610, Mar. 2003.

42. N. Kaiser et al., "Pan-STARRS: A Large Synoptic Survey Telescope Array," in Survey and Other Telescope Technologies and Discoveries. Edited by Tyson, J. Anthony; Wolff, Sidney. Proceedings of the SPIE, Volume 4836, pp. 154-164 (2002)., pp. 154-164, Dec. 2002.

43. B. P. Schmidt, S. C. Keller, P. J. Francis, and M. S. Bessell, "The SkyMapper Telescope and Southern Sky Survey," American Astronomical Society Meeting Abstracts 206, May 2005.

44. S. M. Jefferies and J. C. Christou, "Restoration of Astronomical Images by Iterative Blind Deconvolution," ApJ 415, p. 862, Oct. 1993.

45. E. Diolaiti, O. Bendinelli, D. Bonaccini, L. Close, D. Currie, and G. Parmeggiani, "Analysis of isoplanatic high resolution stellar fields by the StarFinder code," A\&AS 147, pp. 335-346, Dec. 2000.

46. E. Steinbring, S. M. Faber, B. A. Macintosh, D. Gavel, and E. L. Gates, "Characterizing the Adaptive Optics Off-Axis Point-Spread Function. II. Methods for Use in Laser Guide Star Observations," PASP 117, pp. 847-859, Aug. 2005. 